\title{
Mathematical Modeling of Chikungunya Dynamics: Stability and Simulation
}

\author{
Ruchi Arora, Dharmendra Kumar, Ishita Jhamb and Avina Kaur Narang \\ Department of Mathematics, SGTB Khalsa College, \\ University of Delhi, Delhi-11000\%, India \\ ruchi@sgtbkhalsa.du.ac.in, dharmendrakumar@sgtbkhalsa.du.ac.in, \\ ishita.jhamb@gmail.com, kaur.avina45@gmail.com
}

\begin{abstract}
Infection due to Chikungunya virus (CHIKV) has a substantially prolonged recuperation period that is a long period between the stage of infection and recovery. However, so far in the existing models (SIR and SEIR), this period has not been given due attention. Hence for this disease, we have modified the existing SEIR model by introducing a new section of human population which is in the recuperation stage or in other words the human population that is no more showing acute symptoms but is yet to attain complete recovery. A mathematical model is formulated and studied by means of existence and stability of its disease free equilibrium (DFE) and endemic equilibrium (EE) points in terms of the associated basic reproduction number $\left(R_{0}\right)$.
\end{abstract}

\section{RESUMEN}

La infección debida al virus Chikungunya (CHIKV) tiene un período de recuperación sustancialmente prolongado, que es un período largo entre la etapa de infección y recuperación. Sin embargo, hasta ahora en los modelos existentes (SIR y SEIR), este período no ha recibido suficiente atención. Por tanto, para esta enfermedad, hemos modificado el modelo SEIR existente introduciendo una nueva sección de población humana que está en la etapa de recuperación o, en otras palabras, la población humana que ya no muestra síntomas agudos pero todavía no se recupera completamente. Se formula y estudia un modelo matemático a través de la existencia y estabilidad de su equilibrio libre de enfermedad (DFE) y puntos de equilibrio endémico (EE) en términos del número de reproducción básico asociado $\left(R_{0}\right)$.

Keywords and Phrases: Equilibrium point, disease free equilibrium, endemic equilibrium, reproduction number, local stability, global stability.

2020 AMS Mathematics Subject Classification: 92B05, 93A30, 93C15

(cc) BY-NC

(C)2020 by the author. This open access article is licensed under a Creative Commons Attribution-NonCommercial 4.0 International License. 


\section{Introduction}

In recent past, the study of vector borne diseases has gained considerable attention and mathematics have become a useful tool for such studies. Several temporal deterministic models have been proposed for diseases like dengue, malaria, chikungunya etc. Chikungunya is a disease caused by the chikungunya virus, an RNA genome which is a member of the Alphavirus genus in the family of Togaviridae. It is a mosquito borne viral disease which is transmitted to humans through Aedes aegypti mosquito bite [1. In 1952, chikungunya was first confirmed as the cause of an epidemic of dengue like illness on the Comoros islands located on the eastern coast of northern Mozambique 2. Since its discovery, numerous CHIKV outbreaks with irregular intervals of 2-20 years have affected Asian, African, European and American countries. In Thailand, the first report of chikungunya infection occurred in Bangkok in 1958 3]. In India, the virus emerged in parts of Vellore, Calcutta and Maharashtra in the early 1960's [4. The virus continued to spread in Sri Lanka in 1969 and many countries of Southeast Asia such as Myanmar, Indonesia and Vietnam 4. Later, some irregular cases of chikungunya fever were also seen in many provinces of Thailand in the period from 1976 to 1995 [3]. From 1999 to 2000, the reemergence of chikungunya occurred in Democratic Republic of Congo [2], 13,500 cases were reported in Lamu, Kenya in 2004 [5]. In the years 2005-2007, there occurred an outbreak in Reunion islands in the Indian Ocean. In 2007, 197 cases were reported in Europe due to chikungunya [1]. The outbreak mutated to facilitate the disease transmission by Aedes albopictus from the tiger mosquito family. It was a mutation in one of the viral envelope genes which allowed the virus to be present in the mosquito saliva only two days after the infection and seven days in Aedes aegypti mosquitoes. The results indicated that the areas where the tiger mosquitoes are present could have a greater risk of outbreak.

After an effective bite from a mosquito infected with CHIKV, the incubation period (i.e., the time elapsed between exposure to pathogenic organism and when symptoms and signs are first apparent) usually lasts for 3-7 days with fever as the most prominent symptom. The symptoms of chikungunya fever differ from the normal fever as they are accompanied with acute joint pains. Other common symptoms are nausea, rashes, headache and fatigue. Some cases may result in neurological, retinal and carpological complications as well, which makes it difficult for older people to recover as against young people. In some instances, people live with joint pains for years which indicates that the recuperation period can last for a long time. The symptoms of chikungunya are generally mild and the disease may sometimes be misdiagnosed with Zika and Dengue due to similarity in symptoms. There have been very few cases where chikungunya resulted in death and mostly infected individuals are expected to make full recovery with lifelong immunity. As such, there is no preventive vaccine or cure for chikungunya. One can only manage the symptoms by taking medications for temporary relief. To prevent the spread of disease, breeding sites for the mosquitoes should be checked. Using mosquito repellents and wearing long sleeve clothes and full 
pants can help in preventing mosquito bite. For more such information one may refer to [1].

Increasing globalization and factors contributing to climate change brought about a sudden expansion of mosquito breeding sites. This makes it necessary to improve the vector control techniques and to identify the indexes that monitor thresholds for such programs. Through the $20^{\text {th }}$ century, mathematical modeling has been extensively used to study epidemic diseases. Futhermore, this branch of mathematics is also being used to devise optimal control strategies for various infectious diseases. Like M. Barro et al. [6] introduced an optimal control for a SIR model governed by an ODE system with time delay. And, O. K. Oare [7] considered and analyzed a deterministic multipatch hepatitis $\mathrm{C}$ virus model for it.

In context of infection due to chikungunya virus, Y. Dumont et al. 8 proposed a model associated with the time course of the first epidemic of chikungunya in several cities of Reunion Island. A model describing the mosquito population dynamics and the virus transmission to human population was discussed by D. Moulay et al. [9. Although simplistic, L. Yacob et al. [10] gave a model which provided a close approximation of the peak incidence of the outbreak and the final epidemic size. S. Naowarat and I. M. Tang [1] studied the model taking into consideration the presence of two species of Aedes mosquito (Aedes aegypti and Aedes albopictus). D. H. Palacio and J. Ospina [12] derived measures of disease control, by means of three scenarios, namely a single vector, two vectors, and two vectors and human and non-human reservoirs. It also showed the need to periodically evaluate the effectiveness of vector control measures. F. B. Agusto et al. [13 described the chikungunya model of three age structured transmission dynamics by considering juvenile, adult and senior population, where the dynamics of shift in individuals from one stage to another was studied.

In this paper, we introduce a deterministic model to study the dynamics and transmission of chikungunya virus by considering a very significant section from the class of infected individuals. Usually, the existing models focus on the SIR or the SEIR human population model and SEI mosquito population model. Since the period from the infected stage to the complete recovery stage is quite long for this disease, so it becomes significant to study that particular class of human population which has recovered from acute symptoms of the disease but is yet to attain full recovery. Though the class no longer shows the immediate symptoms like fever, rashes, nausea etc. but at the same time they are bearing the latent and the passive effects of the disease like joint pains, fatigue, headache etc. Generally such ailments continue for a prolonged period which may vary from individual to individual. But as long as the patient is suffering from these ailments, he or she cannot be declared as fully recovered [14. Focussing on this category of patients, we introduce a new compartment between compartments of the infected and the recovered human population within the existing SEIR model. We refer to it as the recuperation compartment and denote it by $\mathrm{R}^{\prime}$. So, in this paper our aim is to study, analyse and investigate in detail the model showing the interaction between the human population divided into five compartments resulting 
into a SEIR'R model and the mosquito population into the traditional three compartments which we denote by XYZ model.

The paper is divided as follows: Section 2 deals with the formulation of the model, section 3 analyses its feasibility, section 4 determines the disease free equilibrium (DFE) and establishes its local and global stability, section 5 deals with the existence of endemic equilibrium (EE) and its local stability. Also by means of simulation of the formulated model, we provide a visualization to the dynamics of this disease, in section 6 . Finally related to our model, some conclusions are stated.

\section{Model Formulation}

In this section, an epidemic model is formulated for chikungunya disease. Let $N_{H}$ represent the total human population which is further subdivided into five categories; susceptibles (S), humans exposed to infection (E), infected humans (I), population in recuperation phase $\left(\mathrm{R}^{\prime}\right)$ and finally the population that has attained complete recovery $(\mathrm{R})$. So, the traditional SEIR epidemic model has been modified to a more relevant and practically applicable SEIR ${ }^{\prime} \mathrm{R}$ model. Hence in this case, at any time $t$

$$
N_{H}(t)=S(t)+E(t)+I(t)+R^{\prime}(t)+R(t) .
$$

Let $N_{M}$ represent the total mosquito population which is further subdivided into 3 parts; susceptible mosquitoes (X), mosquitoes exposed to infection (Y) and infectious mosquitoes (Z). So the total mosquito population is $N_{M}(t)=X(t)+Y(t)+Z(t)$.

For human population, let $\mu$ be the constant birth rate and $\zeta$ be the natural death rate. Then the rate of change of susceptible human population is given by

$$
\frac{d S}{d t}=\mu-\lambda_{H} S-\zeta S
$$

where $\lambda_{H}=\frac{\beta B_{H} Z}{N_{H}} . B_{H}$ is the transmission probability per contact for susceptible humans (S) and $\beta$ is the mosquito biting rate for transfer of infection from infectious mosquito class $(\mathrm{Z})$ to susceptible human population (S). As only the susceptible human population out of the whole population is prone to get infection, thereby we divide the expression by $N_{H}$. The rate of change of exposed human population is given by

$$
\frac{d E}{d t}=\lambda_{H} S-\alpha E-\zeta E,
$$

where $\alpha$ is the rate of progression from exposed (E) to infected (I) human population. Here the inflow rate is $\lambda_{H}$ and outflow rate is $\alpha+\zeta$. Similarly, the rate of change of infected human population is 


$$
\frac{d I}{d t}=\alpha E-\gamma I-\left(\zeta+\zeta_{1}\right) I
$$

where $\zeta_{1}$ is death rate due to infection and $\gamma$ is progression rate of infected (I) to recuperated $\left(R^{\prime}\right)$ human population. Now, rate of change of human population in recuperation phase is

$$
\frac{d R^{\prime}}{d t}=\gamma I-\lambda R^{\prime}-\left(\zeta+\zeta_{2}\right) R^{\prime}
$$

where $\zeta_{2}$ is the death rate of humans in recuperated phase due to virus and $\lambda$ is the rate of progression from recuperation $\left(\mathrm{R}^{\prime}\right)$ to the recovery phase $(\mathrm{R})$. Finally, rate of change of recovered human population is,

$$
\frac{d R}{d t}=\lambda R^{\prime}-\zeta R
$$

Again for the mosquito population, let $\rho$ be the constant birth rate and $\kappa$ be the natural death rate, then the rate of susceptible mosquito population is given by

$$
\frac{d X}{d t}=\rho-\lambda_{M} X-\kappa X
$$

where $\lambda_{M}=\frac{\nu B_{M}\left(I+R^{\prime}\right)}{N_{H}} . B_{M}$ is the transmission probability per contact for susceptible mosquito population $(\mathrm{X})$ and $\nu$ is the mosquito biting rate for transfer of infection from infected (I) or recuperated $\left(R^{\prime}\right)$ human population to susceptible mosquito population (X). Again there occurs division by $N_{H}$ because infection can be transfered to mosquitoes only by a certain fraction of human population. Now, the rate of change of exposed mosquito population is given by

$$
\frac{d Y}{d t}=\lambda_{M} X-\psi Y-\kappa Y,
$$

where $\psi$ is the progression rate from exposed (Y) to infectious $(\mathrm{Z})$ mosquito population. Here the inflow rate is $\lambda_{M}$ and outflow rate is $\psi+\kappa$. Similarly, the rate of change of mosquito population carrying infection is

$$
\frac{d Z}{d t}=\psi Y-\kappa Z
$$

Compiling the above discussion, we get the eight dimensional system of nonlinear ordinary differential equations that forms our Chikungunya Model $(\mathrm{CM})$. The parameters and the variables used in the model $(\mathrm{CM})$ are described in Table 1. To get a clear view of the inter relationships between various compartments in discussion, one may refer to Figure 1 which shows the schematic flow 
diagram of the model. The model (CM) is as follows:

(CM)

$$
\begin{aligned}
\frac{d S}{d t} & =\mu-\frac{\beta B_{H} Z S}{N_{H}}-\zeta S, \\
\frac{d E}{d t} & =\frac{\beta B_{H} Z S}{N_{H}}-\alpha E-\zeta E, \\
\frac{d I}{d t} & =\alpha E-\gamma I-\left(\zeta+\zeta_{1}\right) I, \\
\frac{d R^{\prime}}{d t} & =\gamma I-\lambda R^{\prime}-\left(\zeta+\zeta_{2}\right) R^{\prime}, \\
\frac{d R}{d t} & =\lambda R^{\prime}-\zeta R, \\
\frac{d X}{d t} & =\rho-\frac{\nu B_{M}\left(I+R^{\prime}\right) X}{N_{H}}-\kappa X, \\
\frac{d Y}{d t} & =\frac{\nu B_{M}\left(I+R^{\prime}\right) X}{N_{H}}-\psi Y-\kappa Y, \\
\frac{d Z}{d t} & =\psi Y-\kappa Z .
\end{aligned}
$$

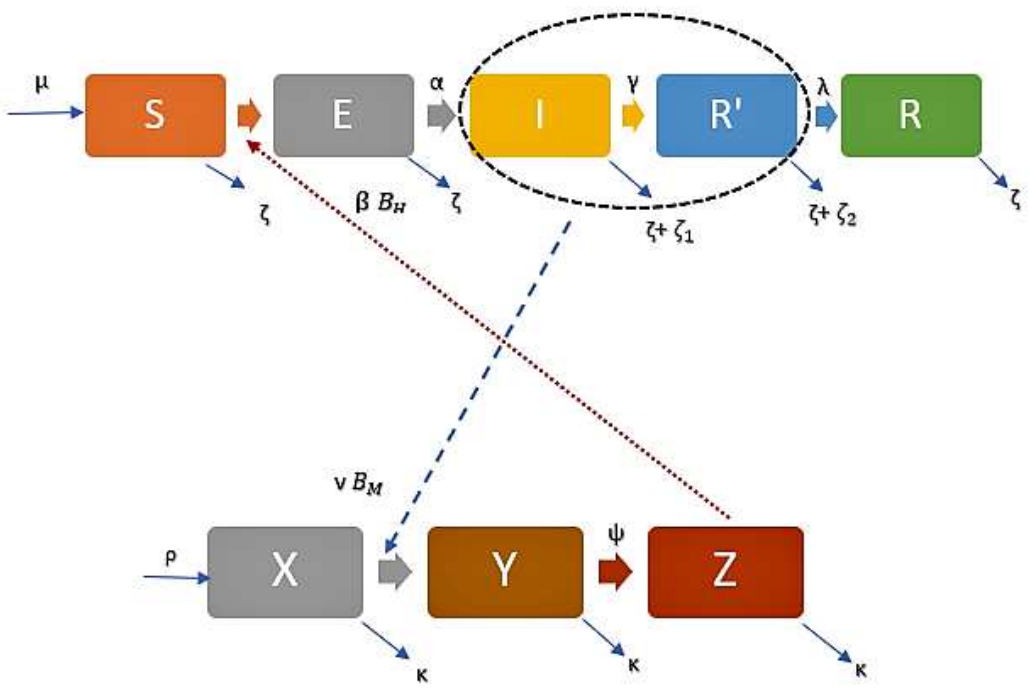

Figure 1: Schematic diagram of Chikungunya Model (CM) 
Table 1: Description of variables and parameters used in model (CM)

\begin{tabular}{|c|l|}
\hline Variables & Description \\
\hline $\mathrm{S}$ & Susceptible human population. \\
\hline $\mathrm{E}$ & $\begin{array}{l}\text { Exposed human population. } \\
\text { (Population that is infected but yet to show symptoms). }\end{array}$ \\
\hline $\mathrm{I}$ & Infected Human population showing symptoms. \\
\hline $\mathrm{R}^{\prime}$ & Human population in recuperation phase. \\
\hline $\mathrm{R}$ & Fully recovered human population. \\
\hline $\mathrm{X}$ & Susceptible mosquito population. \\
\hline $\mathrm{Y}$ & $\begin{array}{l}\text { Exposed mosquito population. } \\
\text { (carrying infection but not yet capable to spread it). }\end{array}$ \\
\hline $\mathrm{Z}$ & Infectious mosquito population spreading the disease. \\
\hline \hline Parameters & Description \\
\hline$\mu$ & Human birth rate. \\
\hline$\beta$ & $\begin{array}{l}\text { Mosquito biting rate for transfer of infection from } \\
\text { infectious mosquito class (Z) to susceptible human population (S). }\end{array}$ \\
\hline$\alpha$ & Progression rate of exposed to infected human population. \\
\hline$\gamma$ & Progression rate of infected to recuperated human population. \\
\hline$\lambda$ & Progression rate of recuperated to fully recovered human population. \\
\hline$\rho$ & Mosquito birth rate. \\
\hline$\nu$ & $\begin{array}{l}\text { Mosquito biting rate for transfer of infection from } \\
\text { infected human population(I) or population under recuperation phase }\left(\mathrm{R}^{\prime}\right) \\
\text { to susceptible mosquito population (X). }\end{array}$ \\
\hline$\psi$ & Progression rate from exposed to infectious mosquito population. \\
\hline$\zeta$ & Natural death rate for human population. \\
\hline$\zeta_{1}$ & Human death rate in infected stage due to viral infection. \\
\hline$\zeta_{2}$ & Human death rate due to infection under recovery phase. \\
\hline$B_{H}$ & Natural death rate for mosquito population. \\
\hline$B_{M}$ & Transmission probability per contact in susceptible humans. \\
\hline$N_{H}$ & Transmission probability per contact in susceptible mosquitoes. \\
\hline & Total human population, i.e. S+E+I+R + R. \\
\hline
\end{tabular}


Table 2: Range of Parameters for the model (CM)

\begin{tabular}{|c|c|c|}
\hline Parameters & Range & References \\
\hline$\mu$ & $400 \times \frac{1}{15 \times 365}-400 \times \frac{1}{12 \times 365}$ & [15, 16 \\
\hline$\beta$ & $0.19-0.39$ & [15, 17] \\
\hline$\alpha$ & $\frac{1}{4}-\frac{1}{2}$ & $44,15,18,19,20,21]$ \\
\hline$\gamma$ & $\frac{1}{4}-\frac{1}{2}$ & Estimated [14] \\
\hline$\lambda$ & $\frac{1}{8}-\frac{1}{4}$ & Estimated [14] \\
\hline$\rho$ & $500 \times 0.015-500 \times 0.33$ & 15, 16, 22, 23. \\
\hline$\nu$ & $0.19-0.39$ & [15, 17] \\
\hline$\psi$ & $\frac{1}{6}-\frac{1}{2}$ & {$[9,18,20,24]$} \\
\hline$\zeta$ & $\frac{1}{60 \times 365}-\frac{1}{18 \times 365}$ & 13 \\
\hline$\zeta_{1}$ & $\frac{1}{10^{5}}-\frac{1}{10^{4}}$ & 25 \\
\hline$\zeta_{2}$ & $\frac{1}{10^{6}}-\frac{1}{10^{5}}$ & 25 \\
\hline$\kappa$ & $\frac{1}{42}-\frac{1}{14}$ & 9, $18,19,20,21$ \\
\hline$B_{H}$ & $0.001-0.54$ & {$[8,15,26,18,27$} \\
\hline$B_{M}$ & $0.005-0.35$ & $8,26,27,28,29$ \\
\hline
\end{tabular}


Table 3: Values of Parameters for Simulation

\begin{tabular}{|c|c|c|}
\hline Parameters & $R_{0}<1$ & $R_{0}>1$ \\
\hline$\mu$ & $400 \times \frac{1}{15 \times 365}$ & $400 \times \frac{1}{15 \times 365}$ \\
\hline$\beta$ & 0.25 & 0.30 \\
\hline$\alpha$ & $\frac{1}{3}$ & $\frac{1}{4}$ \\
\hline$\gamma$ & $\frac{1}{3}$ & $\frac{1}{4}$ \\
\hline$\lambda$ & $\frac{1}{7}$ & $\frac{1}{8}$ \\
\hline$\rho$ & $500 \times 0.1675$ & $500 \times 0.2$ \\
\hline$\nu$ & 0.25 & 0.30 \\
\hline$\psi$ & $\frac{1}{3.5}$ & $\frac{1}{4}$ \\
\hline$\zeta$ & $\frac{1}{40 \times 365}$ & $\frac{1}{30 \times 365}$ \\
\hline$\zeta_{1}$ & $\frac{1}{10^{4}}$ & $\frac{1}{10^{5}}$ \\
\hline$\zeta_{2}$ & $\frac{1}{10^{5}}$ & $\frac{1}{10^{6}}$ \\
\hline$\kappa$ & $\frac{1}{14}$ & $\frac{1}{30}$ \\
\hline$B_{H}$ & 0.24 & 0.30 \\
\hline$B_{M}$ & 0.24 & 0.30 \\
\hline
\end{tabular}




\section{Preliminary Results}

\subsection{Positivity of Solutions}

In order to establish the epidemiological meaningfullness [13, we prove the non negativity of the state variables for the formulated model at all $t>0$.

Theorem 3.1: The solution $M(t)=\left(S, E, I, R^{\prime}, R, X, Y, Z\right)$ of model $(\mathrm{CM})$ with $M(0) \geq 0$, is non negative for all $t>0$. Moreover,

$$
\lim _{t \rightarrow \infty} \sup N_{H}(t)=\frac{\mu}{\zeta} \text { and } \lim _{t \rightarrow \infty} \sup N_{M}(t)=\frac{\rho}{\kappa}
$$

where $N_{H}(t)=S(t)+E(t)+I(t)+R^{\prime}(t)+R(t)$ and $N_{M}(t)=X(t)+Y(t)+Z(t)$.

Proof: Let $t_{1}=\sup \{t>0: M(t)>0\}$. Clearly $t_{1}>0$. Consider the first equation of the model $(\mathrm{CM})$,

$$
\frac{d S}{d t}=\mu-\frac{\beta B_{H} S Z}{N_{H}}-\zeta S .
$$

Solving the differential equation we have,

$$
\begin{gathered}
\frac{d}{d t}\left\{S(t) \exp \left[\left(\int_{0}^{t_{1}} \frac{\beta B_{H} Z(\tau)}{N_{H}(\tau)} d \tau+\zeta t\right)\right]\right\}=\mu \exp \left[\left(\int_{0}^{t_{1}} \frac{\beta B_{H} Z(\tau)}{N_{H}(\tau)} d \tau+\zeta t\right)\right] \\
\Longrightarrow S\left(t_{1}\right) \exp \left[\left(\int_{0}^{t_{1}} \frac{\beta B_{H} Z(\tau)}{N_{H}(\tau)} d \tau+\zeta t_{1}\right)\right]-S(0)=\int_{0}^{t_{1}} \mu \exp \left[\left(\int_{0}^{u} \frac{\beta B_{H} Z(\tau)}{N_{H}(\tau)} d \tau+\zeta u\right)\right] d u .
\end{gathered}
$$

Furthermore,

$$
\begin{aligned}
S\left(t_{1}\right) & =S(0) \exp \left[\left(-\int_{0}^{t_{1}} \frac{\beta B_{H} Z(\tau)}{N_{H}(\tau)} d \tau+\zeta t_{1}\right)\right] \\
& +\exp \left[\left(-\int_{0}^{t_{1}} \frac{\beta B_{H} Z(\tau)}{N_{H}(\tau)} d \tau+\zeta t_{1}\right)\right] \int_{0}^{t_{1}} \mu \exp \left[\left(\int_{0}^{u} \frac{\beta B_{H} Z(\tau)}{N_{H}(\tau)} d \tau+\zeta u\right)\right] d u>0 .
\end{aligned}
$$

Similarly, the non negativity can be shown for all the state variables, i.e., $M\left(t_{1}\right)>0$ and therefore $M(t)>0$ for all $t>0$. In fact, we now have, $0<S(t) \leq N_{H}(t), 0<E(t) \leq N_{H}(t), 0<I(t) \leq$ $N_{H}(t), 0<R^{\prime}(t) \leq N_{H}(t), 0<R(t) \leq N_{H}(t) ; 0<X(t) \leq N_{M}(t), 0<Y(t) \leq N_{M}(t), 0<Z(t) \leq$ $N_{M}(t)$. As the total human population is given by $N_{H}(t)=S(t)+E(t)+I(t)+R^{\prime}(t)+R(t)$, the rate of change of human population with respect to time is given by

$$
\begin{aligned}
\frac{d N_{H}}{d t} & =\mu-\zeta\left(S+E+I+R^{\prime}+R\right)-\zeta_{1} I-\zeta_{2} R^{\prime} \\
& =\mu-\zeta N_{H}-\zeta_{1} I-\zeta_{2} R^{\prime} \\
& \leq \mu-\zeta N_{H} .
\end{aligned}
$$


Now for $N_{M}(t)=X(t)+Y(t)+Z(t)$,

$$
\frac{d N_{M}}{d t} \leq \rho-\kappa N_{M}
$$

Let $N=\frac{\mu}{\zeta}$. As $t \rightarrow \infty$, the disease will disappear. Therefore, $\lim _{t \rightarrow \infty} \sup I(t)=0$ and $\lim _{t \rightarrow \infty} \sup R^{\prime}(t)=$ 0. Now, $\frac{d N_{H}}{d t}=\mu-\zeta N_{H}$ this implies $N_{H}(t)=\frac{\mu}{\zeta}+\left(N_{H}(0)-\frac{\mu}{\zeta}\right) e^{-\zeta t}$, which further implies $\lim _{t \rightarrow \infty} N_{H}(t)=\frac{\mu}{\zeta}=N$. This follows that $0<\lim _{t \rightarrow \infty} \sup N_{H}(t) \leq N=\frac{\mu}{\zeta}$ if $\lim _{t \rightarrow \infty} \sup I(t)=0$ and $\lim _{t \rightarrow \infty} \sup R^{\prime}(t)=0$. And if $N_{H}>N=\frac{\mu}{\zeta}$ then from (3.1),$\frac{d N_{H}}{d t}<0$. Similarly, it can be seen that $0<\lim _{t \rightarrow \infty} \sup N_{M}(t) \leq \frac{\rho}{\kappa}$.

\subsection{Invariant Region}

Consider $\Re=\Re_{H} \times \Re_{M} \subset \mathbb{R}_{+}^{5} \times \mathbb{R}_{+}^{3}$, where

$$
\begin{aligned}
& \Re_{H}=\left\{S, E, I, R^{\prime}, R: N_{H}(t) \leq \frac{\mu}{\zeta}\right\}, \\
& \Re_{M}=\left\{X, Y, Z: N_{M}(t) \leq \frac{\rho}{\kappa}\right\} .
\end{aligned}
$$

Now, we establish the positive invariance [13], of the region $\Re$ associated to the model (CM). That is, we show that solutions in $\Re$ remain in $\Re$ for all $t>0$.

Theorem 3.2: The region $\Re \subset \mathbb{R}_{+}^{8}$ is positively invariant for the model (CM), with non-negative initial conditions in $\mathbb{R}_{+}^{8}$.

Proof : As seen in Theorem 3.1, $\frac{d N_{H}}{d t} \leq \mu-\zeta N_{H}$ and $\frac{d N_{M}}{d t} \leq \rho-\kappa N_{M}$. By using standard comparison theorem [30, it can be seen that, $N_{H}(t) \leq \frac{\mu}{\zeta}=N$. So, clearly every solution in $\Re_{H}$ remains in $\Re_{H}$ for all $t>0$. Similar is the case for every solution of $\Re_{M}$. Hence, the region $\Re$ is positively invariant and contains all solutions of $\mathbb{R}_{+}^{8}$ for model $(\mathrm{CM})$.

In the following sections, we show the existence and stability of the disease free equilibrium (DFE) and endemic equilibrium (EE) for the model (CM).

\section{Disease Free Equilibrium (DFE)}

In this section, we find a unique disease free equilibrium (DFE) for the model (CM) and then analyse its stability. 


\subsection{Existence of Equilibrium}

To determine the disease free equilibrium (DFE) of the model, we consider the sections of populations that are free from disease and put their time derivatives equal to zero. Let DFE be denoted by $E_{d}=\left(S^{*}, E^{*}, I^{*}, R^{*}, R^{*}, X^{*}, Y^{*}, Z^{*}\right)$. As sections of susceptible and recovered humans as well as susceptible mosquitoes are the only sections free from disease therefore $E_{d}=$ $\left(S^{*}, 0,0,0, R^{*}, X^{*}, 0,0\right)$. Solving the differential equations of the model (CM), DFE is obtained as $E_{d}=\left(\frac{\mu}{\zeta}, 0,0,0,0, \frac{\rho}{\kappa}, 0,0\right)$.

\subsection{Reproduction Number}

Let the basic reproduction number be denoted by $R_{0}$, which is defined as the expected number of secondary cases produced by a single (typical) infection in a population that is completely disease free. To find the threshold quantity $R_{0}$ [31, 32, we consider the next generation matrix $G$, which comprises of two matrices $F$ and $V^{-1}$, where $F=\frac{d \mathcal{F}_{i}\left(x_{0}\right)}{d x_{j}}$ and $V=\frac{d \mathcal{V}_{i}\left(x_{0}\right)}{d x_{j}}$ for $1 \leq i, j \leq 5$. Here, $\mathcal{F}_{i}$ represents the new infection, whereas $\mathcal{V}_{i}$ corresponds to the transfers of infection from one compartment to another. Let $x_{0}$ be the disease free equilibrium state. Hence, the reproduction number is the largest eigen value of the next generation matrix $G$ (defined as the product of matrices $F$ and $V^{-1}$ ), that is the largest eigen value of the matrix, $G=F V^{-1}$. Corresponding to the model $(\mathrm{CM})$,

$$
\mathcal{F}=\left[\begin{array}{c}
\frac{\beta B_{H} S Z}{N_{H}} \\
0 \\
0 \\
\frac{\nu B_{M}\left(I+R^{\prime}\right) X}{N_{H}} \\
0
\end{array}\right] \quad \text { and } \quad \mathcal{V}=\left[\begin{array}{c}
\alpha E+\zeta E \\
-\alpha E+\gamma I+\left(\zeta+\zeta_{1}\right) I \\
-\gamma I+\lambda R^{\prime}+\left(\zeta+\zeta_{2}\right) R^{\prime} \\
\psi Y+\kappa Y \\
-\psi Y+\kappa Z
\end{array}\right]
$$

Next, we find the Jacobian $F$ and $V$ of the matrices $\mathcal{F}$ and $\mathcal{V}$ respectively and the eigen values of the matrix $G=F V^{-1}$, gives the reproduction number as

$$
R_{0}=\frac{\sqrt{\rho \nu \psi \zeta \alpha \beta B_{H} B_{M}\left(\lambda+\gamma+\zeta+\zeta_{2}\right)}}{\kappa \sqrt{\mu(\psi+\kappa)(\zeta+\alpha)\left(\zeta+\gamma+\zeta_{1}\right)\left(\zeta+\lambda+\zeta_{2}\right)}} .
$$




\subsection{Local Stability}

Theorem 4.1 : The DFE of the chikungunya model (CM) is locally asymptotically stable, if $R_{0}<1$ and unstable if $R_{0}>1$, where $R_{0}$ is the associated reproduction number.

Proof : We consider the system of non linear differential equations, corresponding to the model $(\mathrm{CM})$ to evaluate its Jacobian matrix. Let $J_{D}$ denote the Jacobian of the system at DFE that is,

$$
J_{D}=\left[\begin{array}{cccccccc}
-\zeta & 0 & 0 & 0 & 0 & 0 & 0 & -B_{H} \beta \\
0 & -\alpha-\zeta & 0 & 0 & 0 & 0 & 0 & B_{H} \beta \\
0 & \alpha & -\gamma-\zeta-\zeta_{1} & 0 & 0 & 0 & 0 & 0 \\
0 & 0 & \gamma & -\lambda-\zeta-\zeta_{2} & 0 & 0 & 0 & 0 \\
0 & 0 & 0 & \lambda & -\zeta & 0 & 0 & 0 \\
0 & 0 & -\frac{\nu B_{M} \rho \zeta}{\kappa \mu} & -\frac{\nu B_{M} \rho \zeta}{\kappa \mu} & 0 & -\kappa & 0 & 0 \\
0 & 0 & \frac{\nu B_{M} \rho \zeta}{\kappa \mu} & \frac{\nu B_{M} \rho \zeta}{\kappa \mu} & 0 & 0 & -\psi-\kappa & 0 \\
0 & 0 & 0 & 0 & 0 & 0 & \psi & -\kappa
\end{array}\right]
$$

Clearly, the trace of the matrix $J_{D}$ is negative and determinant of matrix $J_{D}$ [33, 34], is given by

$$
\operatorname{det}\left(J_{D}\right)=\frac{-\zeta^{2}\left[\kappa^{2} \mu(\psi+\kappa)\left(\zeta(\zeta+\alpha+\gamma)+\alpha \gamma+\zeta \zeta_{1}+\zeta_{1} \alpha\right)\left(-\zeta-\lambda-\zeta_{2}\right)\right]+\rho \nu \zeta \psi \alpha \beta B_{H} B_{M}\left(\zeta+\lambda+\gamma+\zeta_{2}\right)}{\mu} .
$$

For $R_{0}<1$, we have

$$
\sqrt{\rho \nu \psi \zeta \alpha \beta B_{H} B_{M}\left(\zeta+\gamma+\lambda+\zeta_{2}\right)}<\kappa \sqrt{\mu(\psi+\kappa)(\zeta+\alpha)\left(\zeta+\lambda+\zeta_{2}\right)\left(\zeta+\gamma+\zeta_{1}\right)} .
$$

Therefore,

$$
\kappa^{2} \mu(\psi+\kappa)\left(\zeta+\lambda+\zeta_{2}\right)\left(\zeta(\zeta+\alpha+\gamma)+\alpha \gamma+\zeta \zeta_{1}+\zeta_{1} \alpha\right)-\psi\left[\rho \nu \zeta \alpha \beta B_{H} B_{M}\left(\zeta+\lambda+\gamma+\zeta_{2}\right)\right]>0
$$

or $\operatorname{det}\left(J_{D}\right)>0$. Hence, DFE is locally asymptotically stable if $R_{0}<1$.

\subsection{Global Stability}

Consider the feasible region $\Re_{1}=\left\{D \in \Re: S \leq S^{*}, X \leq X^{*}\right\}$ where $D=\left(S, E, I, R^{\prime}, R, X, Y, Z\right)$, $S^{*}$ and $X^{*}$ are the components of DFE $\left(E_{d}\right)$.

Lemma 4.1: The region $\Re_{1}$ is positively invariant for the model $(\mathrm{CM})$. 
Proof: From the first equation of the model (CM),

$$
\begin{aligned}
\frac{d S}{d t} & =\mu-\frac{\beta B_{H} Z S}{N_{H}}-\zeta S \\
& \leq \mu-\zeta S \\
& \leq \zeta\left(\frac{\mu}{\zeta}-S\right) \\
& \leq \zeta\left(S^{*}-S\right) \\
S & \leq S^{*}+\left(S(0)-S^{*}\right) e^{-\zeta t}
\end{aligned}
$$

Thus, if $S^{*}=\frac{\mu}{\zeta}$ for all $t \geq 0$ and $S(0) \leq S^{*}$, then $S \leq S^{*}$ for all $t \geq 0$. Similarly, for

$$
\begin{aligned}
\frac{d X}{d t} & =\rho-\frac{\nu B_{M}\left(I+R^{\prime}\right) X}{N_{H}}-\kappa X \\
& \leq \rho-\kappa X \\
& \leq \kappa\left(X^{*}-X\right) \\
X & \leq X^{*}+\left(X(0)-X^{*}\right) e^{-\kappa t}
\end{aligned}
$$

Thus, if $X^{*}=\frac{\rho}{\kappa}$ for all $t \geq 0$ and $X(0) \leq X^{*}$, then $X \leq X^{*}$ for all $t \geq 0$. Hence, it has been shown that the region $\Re_{1}$ is positively invariant and attracts all solutions in $\Re_{+}^{8}$ for the model (CM).

Now in order to establish the global asymptotic stability of DFE [35], we rewrite the model (CM) as

$$
\left[\begin{array}{c}
\frac{d T_{U}}{d t}=F\left(T_{U}, T_{I}\right) \\
\frac{d T_{I}}{d t}=G\left(T_{U}, T_{I}\right), \quad G\left(T_{U}, 0\right)=0
\end{array}\right]
$$

where $T_{U}=(S, R, X) \in \mathbb{R}^{3}$ and $T_{I}=\left(E, I, R^{\prime}, Y, Z\right) \in \mathbb{R}^{5}$.

Let $E_{D}^{*}=\left(T_{U}^{*}, 0\right)$ be DFE of $(\mathrm{RM})$ where $T_{U}^{*}=\left(\frac{\mu}{\zeta}, 0, \frac{\rho}{\kappa}\right)$. We now state the following two conditions which must be satisfied to guarantee global asymptotic stability:

(H1) For $\frac{d T_{U}}{d t}=F\left(T_{U}, 0\right), T_{U}^{*}$ is globally asymptotically stable.

(H2) $G\left(T_{U}, T_{I}\right)=A T_{I}-\hat{G}\left(T_{U}, T_{I}\right), \hat{G}\left(T_{U}, T_{I}\right) \geq 0,\left(T_{U}, T_{I}\right) \in \Re$ where $A=\frac{\partial G\left(T_{U}^{*}, 0\right)}{\partial T_{I}}$ is an M-matrix which by definition has the off diagonal elements non-negative.

Theorem 4.2: The fixed point $E_{D}^{*}=\left(T_{U}^{*}, 0\right)$ is globally asymptotic stable (g.a.s) equilibrium of (RM) provided that $R_{0}<1$ and that assumptions (H1) and (H2) are satisfied.

Proof: For the system (RM),

$$
\frac{d T_{U}}{d t}=F\left(T_{U}, 0\right)=\left[\begin{array}{c}
\mu-\zeta S \\
0 \\
\rho-\kappa X
\end{array}\right]
$$


We solve the above linear differential system to get the $S(t)=\frac{\mu}{\zeta}+S^{*}(0) e^{-\mu t}, R(t)=0$ and $X(t)=\frac{\rho}{\kappa}+X^{*}(0) e^{-\kappa t}$ which implies $S(t) \rightarrow \frac{\mu}{\zeta}, R(t) \rightarrow 0$ and $X(t) \rightarrow \frac{\rho}{\kappa}$ as $t \rightarrow \infty$.

Therefore, disease free point $T_{U}^{*}$ is a globally asymptotic stable (g.a.s) equilibrium of $\frac{d T_{U}}{d t}=$ $F\left(T_{U}, 0\right)$. Hence (H1) holds. Clearly it can be seen that

$$
G\left(T_{U}, T_{I}\right)=\left[\begin{array}{c}
\frac{\beta B_{H} Z S}{N_{H}}-\alpha E-\zeta E \\
\alpha E-\gamma I-\left(\zeta+\zeta_{1}\right) I \\
\gamma I-\lambda R^{\prime}-\left(\zeta+\zeta_{2}\right) R^{\prime} \\
\frac{\nu B_{M}\left(I+R^{\prime}\right) X}{N_{H}}-\psi Y-\kappa Y \\
\psi Y-\kappa Z
\end{array}\right]
$$

Also from (H2) $G\left(T_{U}, T_{I}\right)=A T_{I}-\hat{G}\left(T_{U}, T_{I}\right)$, where

$$
A=\frac{\partial G\left(T_{U}^{*}, 0\right)}{\partial T_{I}}=\left[\begin{array}{ccccc}
-\alpha-\zeta & 0 & 0 & 0 & \beta B_{H} \\
\alpha & -\gamma-\zeta-\zeta_{1} & 0 & 0 & 0 \\
0 & \gamma & -\lambda-\zeta-\zeta_{2} & 0 & 0 \\
0 & \frac{\nu B_{M} \rho \zeta}{\kappa \mu} & \frac{\nu B_{M} \rho \zeta}{\kappa \mu} & -\psi-\kappa & 0 \\
0 & 0 & 0 & \psi & -\kappa
\end{array}\right]
$$

Therefore,

$$
\frac{\partial G\left(T_{U}^{*}, 0\right)}{\partial T_{I}} T_{I}=\left[\begin{array}{c}
-\alpha E-\zeta E-\beta B_{H} Z \\
\alpha E-\gamma I-\left(\zeta+\zeta_{1}\right) I \\
\gamma I-\lambda R^{\prime}-\left(\zeta+\zeta_{2}\right) R^{\prime} \\
\frac{\nu B_{M} a \zeta}{\kappa \mu}\left(I+R^{\prime}\right)-(\psi+\kappa) Y \\
\psi Y-\kappa Z
\end{array}\right]
$$

In view of $(\mathrm{H} 2), \hat{G}\left(T_{U}, T_{I}\right)=\frac{\partial G\left(T_{U}^{*}, 0\right)}{\partial T_{I}} T_{I}-G\left(T_{U}, T_{I}\right)$ which gives

$$
\hat{G}\left(T_{U}, T_{I}\right)=\left[\begin{array}{c}
\beta B_{H} Z\left(1-\frac{S}{N_{H}}\right) \\
0 \\
0 \\
\nu B_{M}\left(I+R^{\prime}\right)\left[\frac{\rho \zeta}{\mu \kappa}-\frac{X}{N_{H}}\right] \\
0
\end{array}\right] .
$$

Clearly, $\beta B_{H} Z\left(1-\frac{S}{N_{H}}\right) \geq 0$ as $\frac{S}{N_{H}}<1$. Also, $\frac{\kappa X}{\rho} \leq \frac{\zeta N_{H}}{\mu}$ or $\frac{\rho \zeta}{\mu \kappa} \geq \frac{X}{N_{H}} \Rightarrow \frac{X^{*}}{S^{*}} \geq \frac{X}{N_{H}}$ and from Lemma 4.1 we know $X^{*} \geq X$ and $N_{H}^{*}=S^{*} \geq N_{H}$, which implies $\nu B_{M}\left(I+R^{\prime}\right)\left[\frac{\rho \zeta}{\mu \kappa}-\frac{X}{N_{H}}\right] \geq 0$. Therefore, (H2) holds true. Hence, $E_{D}^{*}=\left(T_{U}^{*}, 0\right)$ is globally asymptotically stable in the region $\Re$ whenever $R_{0} \leq 1$.

\section{Endemic Equilibrium}

In this section, we first determine the endemic equilibrium points for the model (CM), establish its existence and then analyse its stability. 


\subsection{Endemic Equilibrium Points}

Let endemic equilibrium points be denoted by $E_{e}=\left(S^{* *}, E^{* *}, I^{* *}, R^{* *}, R^{* *}, X^{* *}, Y^{* *}, Z^{* *}\right)$. The components of $E_{e}$ are obtained by imposing constant solutions in the model $(\mathrm{CM})$ and solving the algebraic equations. By computations, we have

$$
\begin{aligned}
S^{* *} & =\frac{\mu N_{H}}{\zeta N_{H}+Z^{* *} \beta B_{H}}, \\
E^{* *} & =\frac{\beta B_{H} Z^{* *} \mu}{(\alpha+\zeta)\left(\beta B_{H} Z^{* *}+\zeta N_{H}\right)}, \\
I^{* *} & =\frac{\alpha \beta B_{H} Z^{* *} \mu}{\left(\gamma+\zeta+\zeta_{1}\right)(\alpha+\zeta)\left(\beta B_{H} Z^{* *}+\zeta N_{H}\right)}, \\
R^{* *} & =\frac{\alpha \gamma \beta B_{H} Z^{* *} \mu}{\left(\lambda+\zeta+\zeta_{2}\right)\left(\gamma+\zeta+\zeta_{1}\right)(\alpha+\zeta)\left(\beta B_{H} Z^{* *}+\zeta N_{H}\right)}, \\
R^{* *} & =\frac{\lambda \alpha \beta B_{H} Z^{* *} \mu \gamma}{\zeta\left(\lambda+\zeta+\zeta_{2}\right)\left(\gamma+\zeta+\zeta_{1}\right)(\alpha+\zeta)\left(\beta B_{H} Z^{* *}+\zeta N_{H}\right)}, \\
X^{* *} & =\frac{\rho}{\lambda_{M}+\kappa}, \\
Y^{* *} & =\frac{\rho \lambda_{M}}{\left(\lambda_{M}+\kappa\right)(\psi+\kappa)}, \\
Z^{* *} & =\frac{\rho \psi \lambda_{M}}{\kappa\left(\lambda_{M}+\kappa\right)(\psi+\kappa)} .
\end{aligned}
$$

\subsection{Existence and Uniqueness of Endemic Equilibrium $\left(E_{e}\right)$}

Theorem 5.1 : Chikungunya Model (CM) has a unique endemic equilibrium if $R_{0}>1$. As seen in section 2,

$$
\begin{aligned}
\lambda_{M} & =\frac{\nu B_{M}\left(I^{* *}+R^{* *}\right)}{N_{H}} \\
& =\frac{\nu B_{M} \zeta \alpha \beta \mu B_{H} Z^{* *}\left(\zeta+\zeta_{2}+\lambda+\gamma\right)}{\mu\left(\beta B_{H} Z^{* *}+\mu\right)(\alpha+\zeta)\left(\zeta+\zeta_{1}+\gamma\right)\left(\zeta+\zeta_{2}+\lambda\right)} \\
& =\frac{R_{0}^{2} \mu Z^{* *} \kappa^{2}(\psi+\kappa)}{\rho \psi\left(\beta B_{H} Z^{* *}+\mu\right)}
\end{aligned}
$$

Also, $\lambda_{H}=\frac{\beta B_{H} Z^{* *}}{N_{H}}=\frac{\beta B_{H} \rho \psi \lambda_{M}}{\kappa N_{H}\left(\lambda_{M}+\kappa\right)(\psi+\kappa)}$, or equivalently $\lambda_{M}=\frac{\lambda_{H} \mu \kappa^{2}(\psi+\kappa)}{\beta B_{H} \rho \psi \zeta-\mu \kappa(\psi+\kappa) \lambda_{H}}$. 
Equating both values of $\lambda_{M}$, we get the following linear equation in terms of $\lambda_{H}$ :

$$
\lambda_{H}\left(\rho \psi \beta B_{H}+R_{0}^{2} \mu \kappa(\psi+\kappa)\right)=\left(R_{0}^{2}-1\right) \beta B_{H} \rho \psi \zeta
$$

The unique solution to this equation exists and is given by

$$
\lambda_{H}=\frac{\left(R_{0}^{2}-1\right) \beta B_{H} \rho \psi \zeta}{\rho \psi \beta B_{H}+R_{0}^{2} \mu \kappa(\psi+\kappa)},
$$

which is positive if $R_{0}^{2}>1$. This implies $Z^{* *}>0$, for $R_{0}>1$. Hence, unique endemic equilibrium exists for $R_{0}>1$.

\subsection{Local Stability}

Theorem 5.2: The endemic equilibrium of the chikungunya model (CM) is locally asymptotically stable if $R_{0}>1$.

Proof: We evaluate the Jacobian matrix for the system of nonlinear differential equations corresponding to the model $(\mathrm{CM})$. Let $J_{e}$ denote the Jacobian of the system at $E_{e}$ (which exists for $\left.R_{0}>1\right)$. Clearly, $J_{E}=\left(J_{1}, J_{2}, J_{3}, J_{4}, J_{5}, J_{6}, J_{7}, J_{8}\right)^{\mathrm{T}}$ where

$$
\begin{aligned}
& J_{1}=\left(\frac{-\beta B_{H} Z^{* *}}{N_{H}}+\frac{\beta B_{H} Z^{* *} S^{* *}}{\left(N_{H}\right)^{2}}-\zeta, \frac{\beta B_{H} Z^{* *} S^{* *}}{\left(N_{H}\right)^{2}}, \frac{\beta B_{H} Z^{* *} S^{* *}}{\left(N_{H}\right)^{2}}, \frac{\beta B_{H} Z^{* *} S^{* *}}{\left(N_{H}\right)^{2}}, \frac{\beta B_{H} Z^{* *} S^{* *}}{\left(N_{H}\right)^{2}}, 0,0, \frac{-\beta B_{H} S^{* *}}{N_{H}}\right), \\
& J_{2}=\left(\frac{\beta B_{H} Z^{* *}}{N_{H}}-\frac{\beta B_{H} Z^{* *} S^{* *}}{\left(N_{H}\right)^{2}}, \frac{-\beta B_{H} Z^{* *} S^{* *}}{\left(N_{H}\right)^{2}}-\alpha-\zeta, \frac{-\beta B_{H} Z^{* *} S^{* *}}{\left(N_{H}\right)^{2}}, \frac{-\beta B_{H} Z^{* *} S^{* *}}{\left(N_{H}\right)^{2}}, \frac{-\beta B_{H} Z^{* *} S^{* *}}{\left(N_{H}\right)^{2}}, 0,0, \frac{\beta B_{H} S^{* *}}{N_{H}}\right), \\
& J_{3}=\left(0, \alpha,-\gamma-\zeta-\zeta_{1}, 0,0,0,0,0\right), J_{4}=\left(0,0, \gamma,-\lambda-\zeta-\zeta_{2}, 0,0,0,0\right), \\
& J_{5}=(0,0,0, \lambda,-\zeta, 0,0,0), \\
& J_{6}=\left(\frac{\nu B_{M}\left(I^{* *}+R^{\prime * *}\right) X^{* *}}{\left(N_{H}\right)^{2}}, \frac{\nu B_{M}\left(I^{* *}+R^{\prime * *}\right) X^{* *}}{\left(N_{H}\right)^{2}}, \frac{\nu B_{M}\left(I^{* *}+R^{\prime * *}\right) X^{* *}}{\left(N_{H}\right)^{2}}-\frac{\nu B_{M} X^{* *}}{N_{H}}, \frac{\nu B_{M}\left(I^{* *}+R^{\prime * *}\right) X^{* *}}{\left(N_{H}\right)^{2}}-\frac{\nu B_{M} X^{* *}}{N_{H}},\right. \\
& \left.0,-\frac{\nu B_{M}\left(I^{* *}+R^{\prime * *}\right)}{N_{H}}-\kappa, 0,0\right), \quad \\
& J_{7}=\left(\frac{-\nu B_{M}\left(I^{* *}+R^{\prime * *}\right) X^{* *}}{\left(N_{H}\right)^{2}}, \frac{-\nu B_{M}\left(I^{* *}+R^{\prime * *}\right) X^{* *}}{\left(N_{H}\right)^{2}}, \frac{-\nu B_{M}\left(I^{* *}+R^{\prime * *}\right) X^{* *}}{\left(N_{H}\right)^{2}}+\frac{\nu B_{M} X^{* *}}{N_{H}}, \frac{-\nu B_{M}\left(I^{* *}+R^{\prime * *}\right) X^{* *}}{\left(N_{H}\right)^{2}}+\frac{\nu B_{M} X^{* *}}{N_{H}},\right. \\
& \left.0, \frac{\nu B_{M}\left(I^{* *}+R^{\prime * *}\right)}{N_{H}},-\kappa-\psi, 0\right), J_{8}=(0,0,0,0,0,0, \psi,-\kappa)
\end{aligned}
$$

Further, we reduce $J_{E}$ to the following upper triangular matrix $\left(U_{E}\right) . U_{E}=\left(U_{1}, U_{2}, U_{3}, U_{4}, U_{5}, U_{6}, U_{7}, U_{8}\right)^{\mathrm{T}}$ where

$$
\begin{aligned}
& U_{1}=\left(\frac{-\beta B_{H} Z^{* *}}{N_{H}}+\frac{\beta B_{H} Z^{* *} S^{* *}}{\left(N_{H}\right)^{2}}-\zeta, \frac{\beta B_{H} Z^{* *} S^{* *}}{\left(N_{H}\right)^{2}}, \frac{\beta B_{H} Z^{* *} S^{* *}}{\left(N_{H}\right)^{2}}, \frac{\beta B_{H} Z^{* *} S^{* *}}{\left(N_{H}\right)^{2}}, \frac{\beta B_{H} Z^{* *} S^{* *}}{\left(N_{H}\right)^{2}}, 0,0, \frac{-\beta B_{H} S^{* *}}{N_{H}}\right), \\
& U_{2}=\left(0, \frac{-\beta B_{H} Z^{* *} S^{* *}}{\left(N_{H}\right)^{2}}-\alpha-\zeta, \frac{-\beta B_{H} Z^{* *} S^{* *}}{\left(N_{H}\right)^{2}}, \frac{-\beta B_{H} Z^{* *} S^{* *}}{\left(N_{H}\right)^{2}}, \frac{-\beta B_{H} Z^{* *} S^{* *}}{\left(N_{H}\right)^{2}}, 0,0, \frac{\beta B_{H} S^{* *}}{N_{H}}\right), \\
& U_{3}=\left(0,0,-\gamma-\zeta-\zeta_{1}, 0,0,0,0,0\right), U_{4}=\left(0,0,0,-\lambda-\zeta-\zeta_{2}, 0,0,0,0\right), \\
& U_{5}=(0,0,0,0,-\zeta, 0,0,0), U_{6}=\left(0,0,0,0,0,-\frac{\nu B_{M}\left(I^{* *}+R^{\prime * *}\right)}{N_{H}}-\kappa, 0,0\right), \\
& U_{7}=(0,0,0,0,0,0,-\kappa-\psi, 0), U_{8}=(0,0,0,0,0,0,0,-\kappa)
\end{aligned}
$$


Attached are the eigen values of $U_{E}$ :

$\left(-\zeta,-\kappa,-\psi-\kappa,-\gamma-\zeta-\zeta_{1},-\lambda-\zeta-\zeta_{2},-\frac{\nu B_{M}\left(I^{* *}+R^{* * *}\right)}{N_{H}}-\kappa, \frac{Z^{* *} \beta B_{H}\left(S^{* *}-N_{H}\right)}{\left(N_{H}\right)^{2}}-\zeta, \frac{-\beta B_{H} Z^{* *} S^{* *}}{\left(N_{H}\right)^{2}}-\alpha-\zeta\right)$ each of which is negative and by the criterion given in [36], the endemic equilibrium point $\left(E_{e}\right)$ is locally asymptotically stable if $R_{0}>1$.

\section{Numerical Simulation}

The values of parameters that would be used for simulation of the model (CM) are listed in Table 3. The values used for simulation are taken with reference to their ranges, as stated in Table 2.

Fig. 2a and Fig. 2b are visualizations of the existence and stability of equilibria for the cases, $R_{0}<1$ and $R_{0}>1$, respectively. Also, it illustrates that for $R_{0}<1$, the infection dies out over a period of time as it is the case of DFE. However, in the same time period, it can been seen that the infection continues to persist in the population when $R_{0}>1$ as it is the case of EE.

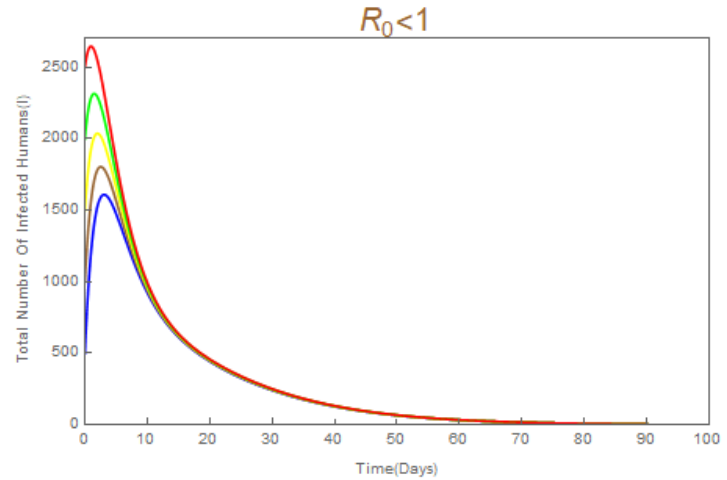

(a)

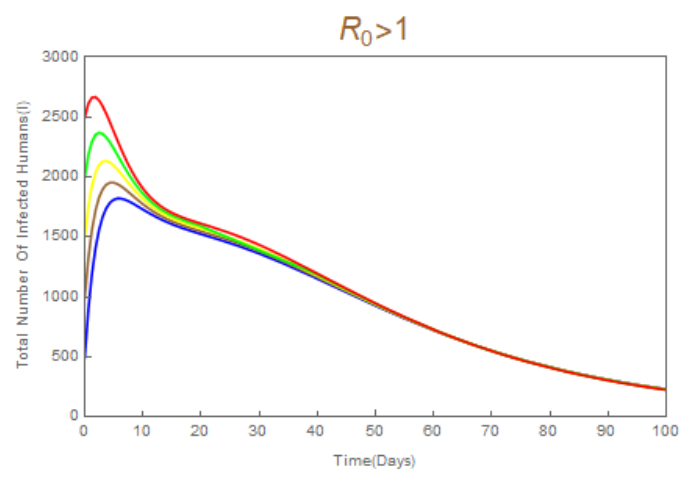

(b)

Figure 2: Total number of Infected Humans (I) with respect to time.

In Fig. [3a, it is clear that the recuperated population ultimately falls down to zero for the case when $R_{0}<1$, where finally the disease dies out and ultimately the entire population will shift to the recovered section with no more inflow into the recuperated part. In contrast, for the same time period, if $R_{0}>1$ (Fig. $3 \mathrm{~b}$ ), the disease persists in the population. Therefore, we can see a substantial proportion of population which is still in the recuperated phase.

Fig. 4 and Fig. 5, both show the time duration around which the number of infected population comes to a fall which is actually the same for recuperated population to reach the peak.

In Fig. 6a, again for $R_{0}<1$, as the disease dies out so it is evidently a situation when the population of the infectious mosquitoes dies out. In contrast to it, for $R_{0}>1$ (Fig. 6b), the number 


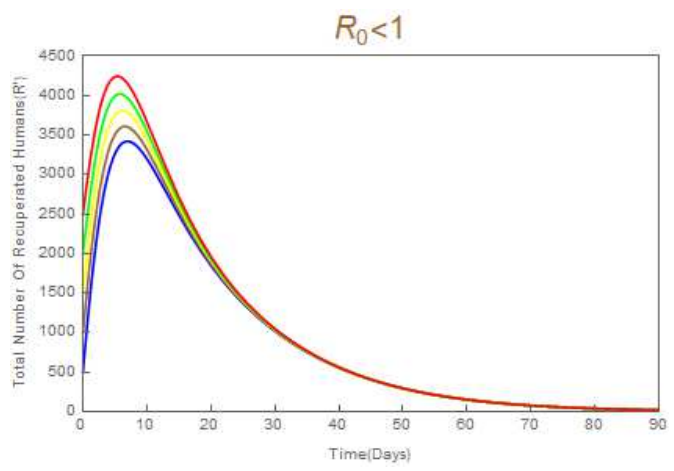

(a)

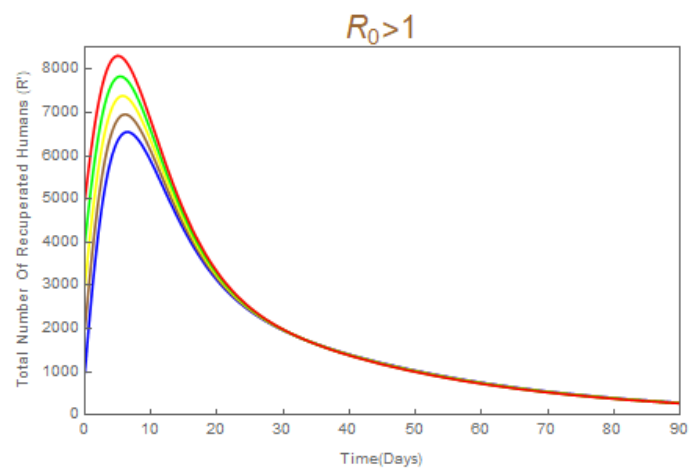

(b)

Figure 3: Total number of Recuperated Humans $\left(R^{\prime}\right)$ with respect to time.

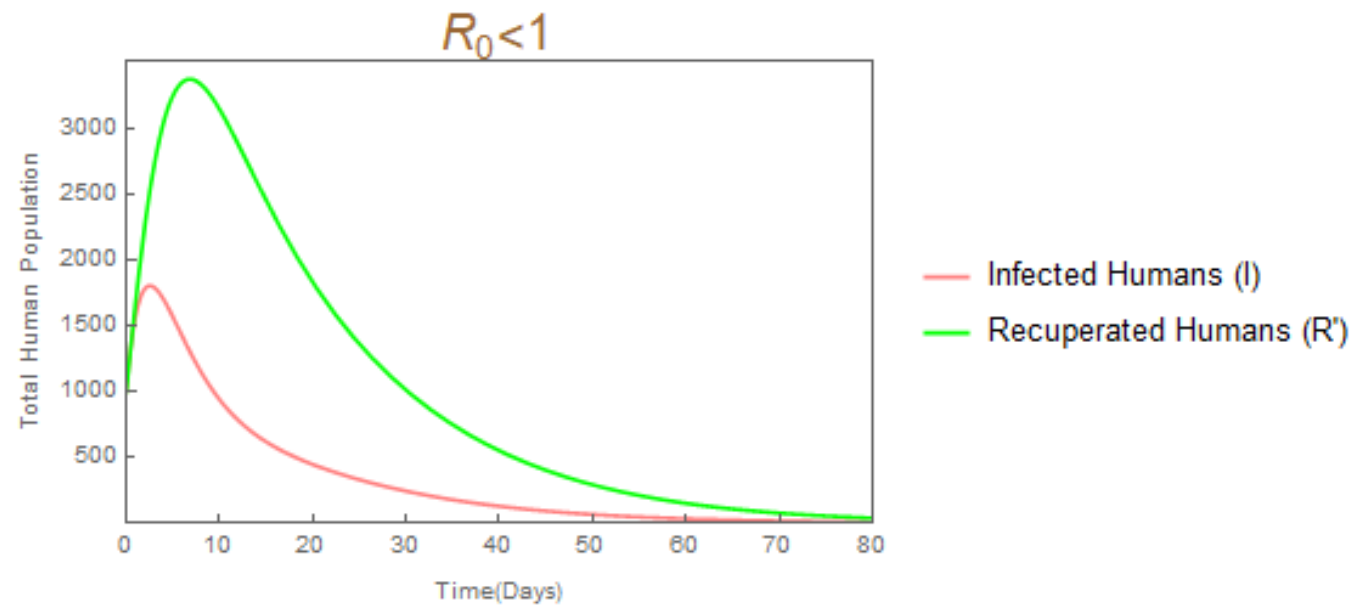

Figure 4: Total number of Infected (I) and Recuperated $\left(R^{\prime}\right)$ Humans when $R_{0}<1$.

of infectious mosquitoes continue to persist in population as it is the case of endemic equilibrium (EE).

Fig. 7 shows the change in the number of infected, recuperated and recovered population with respect to time in accordance with model $(\mathrm{CM})$ whereas Fig. 8 is a simulation of the model $(\mathrm{CM})$ without recuperated section of population.

The curve representing the recovered population in Fig. 8, is an increasing curve showing a rapid increase in the number of people attaining full recovery. But this does not fit in accordance to the case of Chikungunya infection. However, in Fig. 7, we can see the convexity of the curve representing recovered population for a substantial period of time and this is because of the presence of recuperation factor which has been considered in our model. During this period, the recuperation curve is rising higher which is practically more relevant and well in consensus with the nature of this particular disease. 


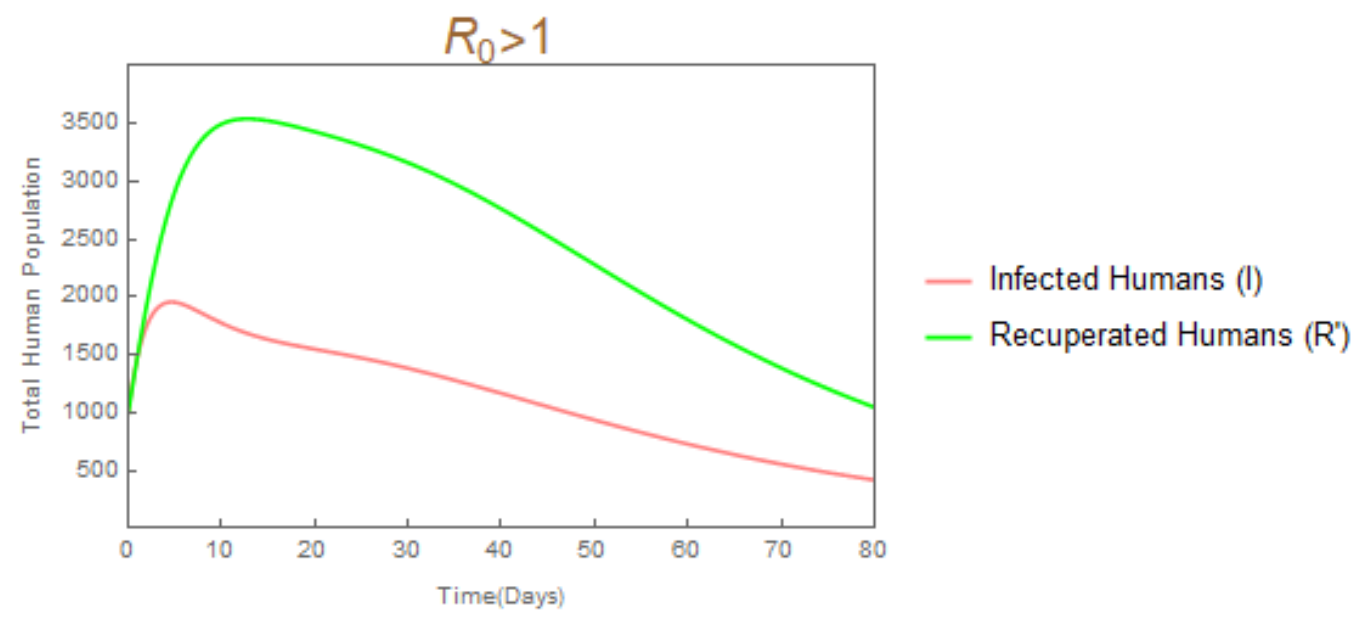

Figure 5: Total number of Infected (I) and Recuperated $\left(R^{\prime}\right)$ Humans when $R_{0}>1$.

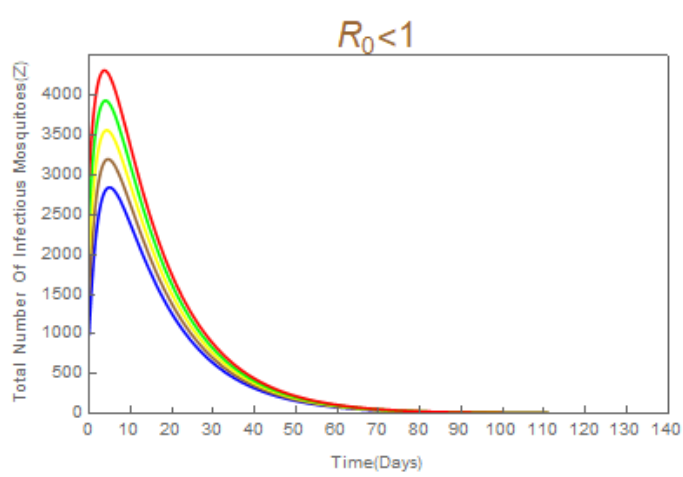

(a)

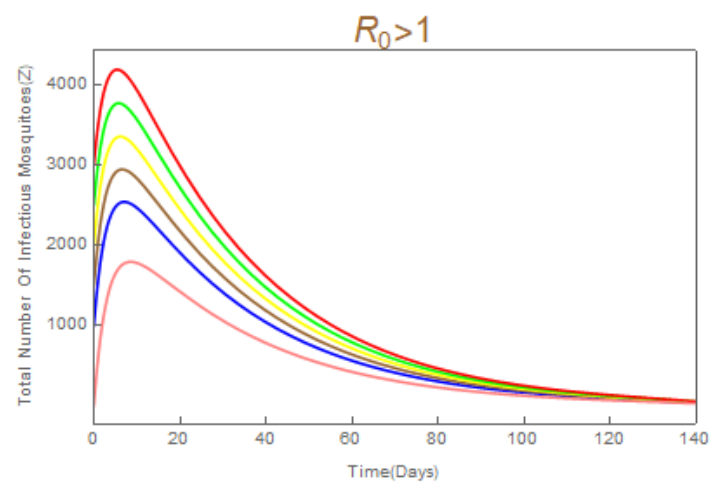

(b)

Figure 6: Total number of Infectious Mosquitoes (Z) with respect to time.

\section{Conclusion}

In this paper, a new deterministic model is formulated to study the transmission dynamics of Chikungunya virus (CHIKV). Making a considerable refinement to the existing models present in the literature, a so far neglected section of human population is introduced, namely the population in the recuperation phase. The study shows that the disease free equilibrium (DFE) of the model is locally as well as globally asymptotically stable whenever existence of an associated reproduction number $R_{0}$, is less than 1 and unstable otherwise. Also, an endemic equilibrium (EE) exists whenever $R_{0}$ is greater than 1 and is locally asymptotically stable too. Simulations of the model make it evident that introduction of the said compartment is well justified, as this model provides a more realistic illustration for Chikungunya infection wherein the quantitative behaviour of disease has given a better visualisation. Moreover, the qualitative behaviour of the disease as studied by 


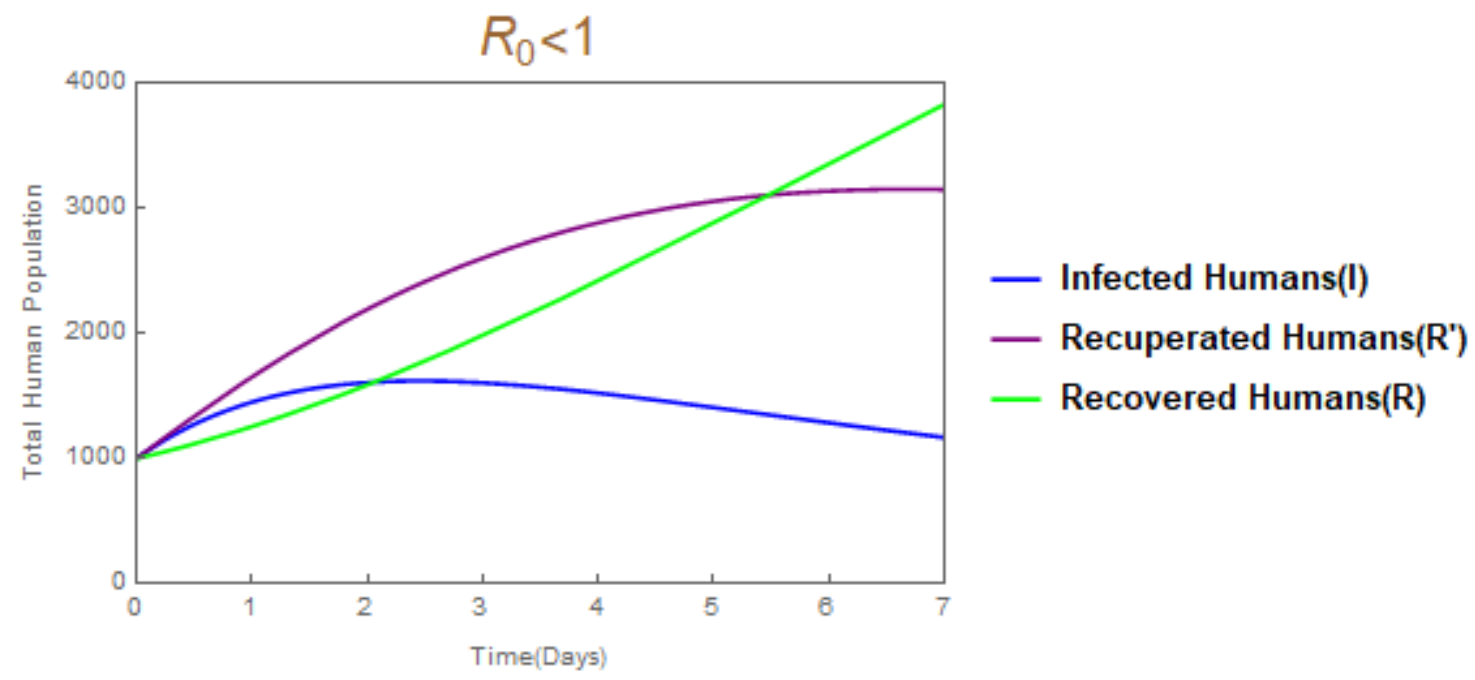

Figure 7: Variation of Infected, Recuperated and Recovered Human Population with time for model $(C M)$.

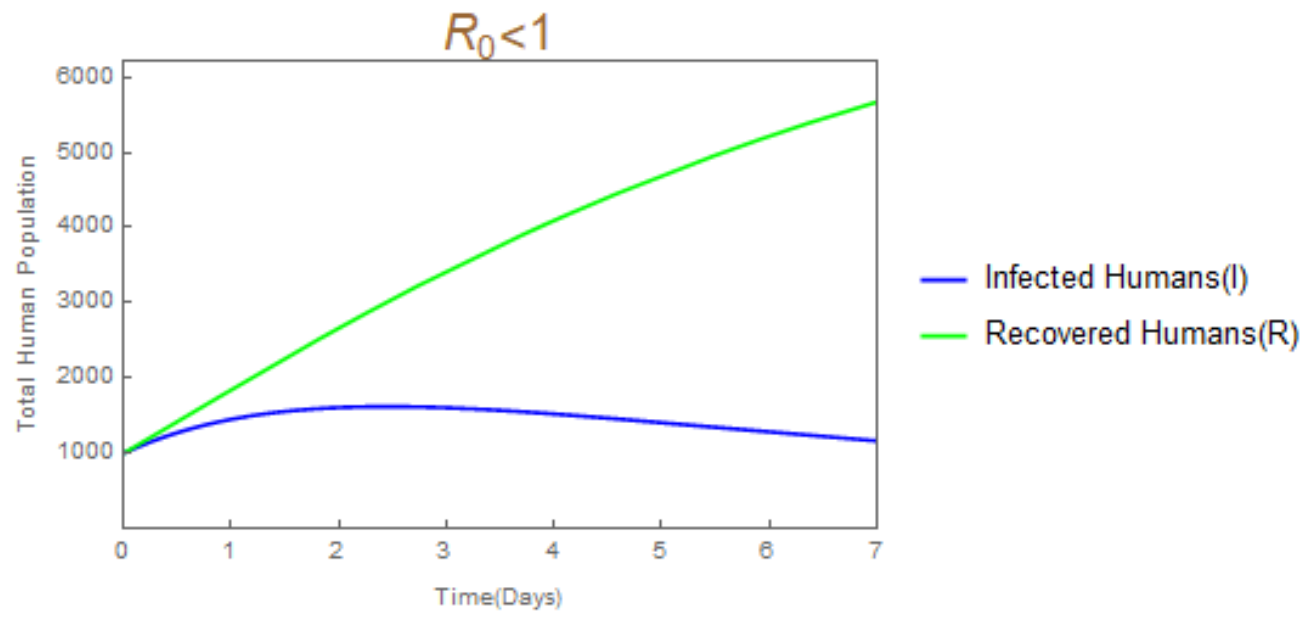

Figure 8: Variation of Infected and Recovered Human Population with time for model (CM) without recuperation section.

various researchers in [14] is very well taken into consideration through our model.

If we do not consider the recuperation section in model (CM), then the following model becomes a special case of our model. It is clearly seen that our model (CM) gives a better illustration to the dynamics of the Chikungunya virus and hence, the proposed model is indeed 
more realistic and practical.

$$
\begin{aligned}
\frac{d S}{d t} & =\mu-\frac{\beta B_{H} Z S}{N_{H}}-\zeta S \\
\frac{d E}{d t} & =\frac{\beta B_{H} Z S}{N_{H}}-\alpha E-\zeta E \\
\frac{d I}{d t} & =\alpha E-\gamma I-\left(\zeta+\zeta_{1}\right) I \\
\frac{d R}{d t} & =\gamma I-\zeta R \\
\frac{d X}{d t} & =\rho-\frac{\nu B_{M} I X}{N_{H}}-\kappa X, \\
\frac{d Y}{d t} & =\frac{\nu B_{M} I X}{N_{H}}-\psi Y-\kappa Y, \\
\frac{d Z}{d t} & =\psi Y-\kappa Z, \quad \text { where } \quad N_{H}(t)=S(t)+E(t)+I(t)+R(t) .
\end{aligned}
$$

Comparison of the above model with our model (CM) is done in section 6 with the help of the graphs shown in Fig. 7 and Fig. 8

\section{Acknowledgement}

The authors are thankful to Dr. Sukhanta Dutta, Dr. Harsha Kharbanda and Tanvi for their valuable suggestions and inputs. This research was supported and funded by the Science Centre, SGTB Khalsa College (Project Code: SGTBKC/SC/SP/2017/08/518). 


\section{References}

[1] WHO. Chikungunya. http://www.who.int/denguecontrol/arbo-viral/other_aborvial_chikun gunya/en/, 2014.

[2] WHO. Chikungunya. http://www.who.int/mediacentre/factsheets/fs327/en/, 2014.

[3] National Center for Biotechnology information. Chikungunya outbreak. http://www.ncbl.nlm.nih.gov/pmc/atricles/PMC4111161, 2014.

[4] G. Pialoux, B. Gäuzère, S. Jauréguiberry, and M. Strobel, Chikungunya, an epidemic arbovirosis. The Lancet infectious diseases, 7:319-27, 062007.

[5] K. Sergon, C. Njuguna, R. Kalani, V. Ofula, C. Onyango, L. Konongoi, S. Bedno, H. Burke, A. M. Dumilla, J. Konde, M. Kariuki Njenga, R. Sang, and R. Breiman. Seroprevalence of chikungunya virus (chikv) infection on lamu island, kenya, october 2004. The American journal of tropical medicine and hygiene, 78:333-7, 032008.

[6] M. Barro, A. Guiro and D. Ouedraogo, Optimal control of a SIR epidemic model with general incidence function and a time delays, Cubo 20 (2018), no. 2, 53-66.

[7] O.K. Oare, Impact and optimal control of movement on a multipatch hepatitis C virus model, TWMS J. Pure Appl. Math. 5 (2014), no. 1, 80-95.

[8] Y. Dumont, F. Chiroleu and C. Domerg, On a temporal model for the Chikungunya disease: modeling, theory and numerics, Math. Biosci. 213 (2008), no. 1, 80-91.

[9] D. Moulay, M. A. Aziz-Alaoui and M. Cadivel, The chikungunya disease: modeling, vector and transmission global dynamics, Math. Biosci. 229 (2011), no. 1, 50-63.

[10] L. Yakob and A. C. A. Clements. A mathematical model of chikungunya dynamics and control: The major epidemic on runion island. PloS one, 8:e57448, 032013.

[11] S. Naowarat and I. M. Tang. Transmission model of chikungunya fever in the presence of two species of aedes mosquitoes. American Journal of Applied Sciences, 10:449-459, 052013.

[12] D. Hincapie-Palacio and J. Ospina. Mathematical modeling of chikungunya fever control. page 94870Z, 052015.

[13] F. B. Agusto et al., Mathematical model of three age-structured transmission dynamics of Chikungunya virus, Comput. Math. Methods Med. 2016, Art. ID 4320514, 31 pp.

[14] A. Mohan, D. H. N. Kiran, I. Chiranjeevi Manohar, and D. Prabath Kumar. Epidemiology, clinical manifestations, and diagnosis of chikungunya fever: Lessons learned from the reemerging epidemic. Indian journal of dermatology, 55:54-63, 012010. 
[15] C. Manore, K. Hickmann, S. Xu, H. Wearing, and J. Hyman. Comparing dengue and chikungunya emergence and endemic transmission in a. aegypti and a. albopictus. Journal of Theoretical Biology, 356:174191, 092014.

[16] N. Chitnis, J. M. Hyman and J. M. Cushing, Determining important parameters in the spread of malaria through the sensitivity analysis of a mathematical model, Bull. Math. Biol. 70 (2008), no. 5, 1272-1296.

[17] H. Delatte, G. Gimonneau, A. Triboire, and D. Fontenille. Influence of temperature on immature development, survival, longevity, fecundity, and gonotrophic cycles of aedes albopictus, vector of chikungunya and dengue in the indian ocean. Journal of medical entomology, 46:3341, 022009 .

[18] Y. Dumont and F. Chiroleu, Vector control for the Chikungunya disease, Math. Biosci. Eng. 7 (2010), no. $2,313-345$.

[19] C. Lahariya and S. Pradhan. Emergence of chikungunya virus in indian subcontinent after 32 years: A review. Journal of vector borne diseases, 43:151-60, 012007.

[20] M. R. Sebastian, R. Lodha, and S. Kabra. Chikungunya infection in children. The Indian Journal of Pediatrics, 76:185-89, 032009.

[21] O. Schwartz and M. Albert. Biology and pathogenesis of chikungunya virus. nat rev microbiol 8:491-500. Nature reviews. Microbiology, 8:491-500, 072010.

[22] K. Costanzo, K. Mormann, and S. Juliano. Asymmetrical competition and patterns of abundance of aedes albopictus and culex pipiens (diptera: Culicidae). Journal of medical entomology, 42:559-70, 082005.

[23] N. A. Hashim, A. Hassan, O. N. Abu Tahir, M. Salmah, and N. Basari. Population analysis of aedes albopictus (skuse) (diptera:culicidae) under uncontrolled laboratory conditions. Tropical biomedicine, 25:117-25, 092008.

[24] M. Dubrulle, L. Mousson, S. Moutailler, M. Vazeille, and A. B. Failloux. Chikungunya virus and aedes mosquitoes: Saliva is infectious as soon as two days after oral infection. PloS one, 4:e5895, 022009.

[25] D. Mavalankar, P. Shastri, T. Bandyopadhyay, J. Parmar, and K. Ramani. Increased mortality rate associated with chikungunya epidemic, ahmedabad, india. Emerging infectious diseases, 14:412-5, 042008.

[26] P. Poletti, G. Messeri, M. Ajelli, R. Vallorani, C. Rizzo, and S. Merler. Transmission potential of chikungunya virus and control measures: The case of italy. PloS one, 6:e18860, 052011. 
[27] M. Turell, J. R. Beaman, and R. F. Tammariello. Susceptibility of selected strains of aedes aegypti and aedes albopictus (diptera: Culicidae) to chikungunya virus. Journal of medical entomology, 29:49-53, 021992.

[28] E. Massad, S. Ma, M. Burattini, Y. Tun, F. Coutinho, and L. Ang. The risk of chikungunya fever in a dengueendemic area. Journal of travel medicine, 15:147-55, 052008.

[29] K. Pesko, C. J. Westbrook, C. Mores, L. Philip Lounibos, and M. Reiskind. Effects of infectious virus dose and bloodmeal delivery method on susceptibility of aedes aegypti and aedes albopictus to chikungunya virus. Journal of medical entomology, 46:395-9, 042009.

[30] V. Lakshmikantham, S. Leela, and A.A. Martynyuk. Stability Analysis of Nonlinear Systems. Systems \& Control: Foundations \& Applications. Springer International Publishing, 2015.

[31] P. van den Driessche and J. Watmough, Reproduction numbers and sub-threshold endemic equilibria for compartmental models of disease transmission, Math. Biosci. 180 (2002), 29-48.

[32] J. H. Jones. Notes on r0. Department of Anthropological Sciences Stanford University, 2007.

[33] G.F. Simmons. Differential Equations with Applications and Historical Notes. Textbooks in Mathematics. CRC Press, 2016.

[34] C. Bhunu, W. Garira, and Z. Mukandavire. Modeling hiv/aids and tuberculosis coinfection. Bulletin of mathematical biology, 71:1745-80, 062009.

[35] C. Castillo-Chavez, Z. Feng and W. Huang, "On the Computation of R0 and Its Role on Global Stability," In: Mathematical Approaches for Emerging and Reemerging Infectious Diseases: An Introduction, Springer-Verlag, New York, 2002, pp. 229-250.

[36] EX. DeJesus and C. Kaufman. Routh-hurwitz criterion in the examination of eigenvalues of a system of nonlinear ordinary differential equations. Physical review. A, 35:5288-5290, 07 1987. 